\title{
Numerical Analysis and Study on Land Subsidence of Tunnel
}

\section{Construction}

\author{
Hezhi Li and Fanghua Liu \\ Nanchang Key Laboratory of Material and Structure Detection, Jiangxi University of Technology
}

Keywords: Tunnel Construction; Land Subsidence; Numerical Analysis; Constitutive model of Soil; ADINA Software; Settlement Trough

\begin{abstract}
With the development of economy, the urban population has increased dramatically; in the process of urban construction, more and more tunnels are built in order to facilitate people's life. Tunnel construction will inevitably cause land subsidence; the buildings on the ground and the normal use of underground pipelines will be affected when the subsidence reaches to a certain extent. The land subsidence has to be predicted in order to reduce the bad influence on the surrounding environment; first, study the theory of land subsidence due to tunnel excavation, and establish the finite element numerical simulation model for land subsidence caused by tunnel construction; then, make comparative analysis on the land subsidence caused by tunnel construction under the two different conditions of no drainage and drainage; finally, make comparative analysis on the main factors causing land subsidence of tunnels and confirm the influence degree of each factor. Through the simulation analysis, present the finite element simulation model and correlative conditions of tunnel construction on the ADINA platform, and find out the range and extent of influence from different factors on land subsidence, which is of great significance for controlling land subsidence, protecting buildings on the ground and underground pipelines in the process of construction.
\end{abstract}

\section{Introduction}

With the rapid economic development and booming urban population, there are nearly 50 cities that have a population more than one million among more than 600 cities in China, and it is imperative to develop rail transit in these cities due to traffic congestion. The cities with rail transit now include Beijing, Tianjin, Shanghai, Changchun, Guangzhou, Dalian, Nanjing, Wuhan, etc. There are nearly 30 cities that are planning subway, and these cities include Shenzhen, Wuhan, Hangzhou, Chengdu, Shenyang, Harbin, Xi 'an, Suzhou, Qingdao, Anshan, etc.

In the process of development and utilization of underground space, the tunnel engineering is born at the right moment. The tunnel project mainly includes the underground railway and highway tunnels built to solve the problems of urban traffic congestion, or various underground passages passing through obstacles; various municipal underground engineering, such as sewage pipes, cable channel as well as municipal pipeline public tunnel (utility tunnel); civil air defense facilities, industrial and civil aspect; underground facilities for culture, sports, entertainment and so on.

As is known to all, the tunnel construction in the cities with dense population and building facilities will inevitably disturb the rock and earth mass, cause the deformation of rock and earth mass and then affect the surface structures and existing pipeline facilities. Land subsidence caused by tunnel construction, especially the tunnel construction with dense surface structures has been the 
topics concerned by researchers at home and abroad. The normal use of surface structures and underground pipelines will be affected when the land subsidence reaches to a certain extent. The land subsidence has to be predicted in order to reduce the damages to the ground buildings and underground facilities as well as harmful effect to the surrounding environment due to the land subsidence caused by tunnel construction. Therefore, the study on ground movement and deformation caused by tunnel excavation is of great significance to surface environment protection and in reducing the harmful effect on existing ground and underground facilities caused by excavation.

Through the finite element method to predict the land subsidence caused by tunnel construction, the land subsidence is treated as mechanical process, which can not only calculate the degree of land subsidence, but also get the stress and deformation condition of inner stratum. According to the stratum condition and the tunnel construction condition, stratum can be assumed as different types of media, such as elasticity, elastic-plastic or visco-elasticity plasticity, etc. Elasticity media finite element method is generally applicable in better stratum and construction condition. Elasticity finite element method was adopted to estimate the land subsidence caused by tunnel construction in Osaka of Japan, and achieved fairly satisfactory results. In the process of tunnel construction, surrounding soils may be disturbed greatly. The estimated value of elastic media analysis of finite element is relatively small, so the soil is generally considered as elastic-plastic media for nonlinear analysis.

Based on the ADINA nonlinear analysis software developed by Professor K. J. Bathe of MIT, the paper made the numerical simulation of soil analysis, and the main analysis methods are as follows:

(1) Consider the disturbance in tunnel excavation, study the range and degree of influence on land subsidence caused by tunnel construction, and set up the finite element numerical simulation model;

(2) Makeuse of all kinds of constitutive model of soil provided by ADINA (Cam-Clay model, Mohr Coloumb model, Drucker Prager model, etc.), make the respective numerical simulation analysis and conduct the second ary development of Duncan-Chang model and comparative study on the basis;

(3) By changing the boundary conditions, parameters of soil and tunnel buried depth in the model, analyze the influencing factors of land subsidence.

Theoretical study on numerical analysis

Finite element is a kind of numerical calculation tool, which is rapidly developed from the mid of 1950s to the end of 1960s. The theory was in the primary stage at that time, and the hardware and software of computer could not meet the demand; finite element method and finite element program could not be popularized in engineering. Large general finite element program was developed in the late 1960s and early 1970s, and the program became powerful analysis tool in engineering because it has strong functions, convenience, reliable calculation results and high efficiency. At present, the finite element method plays an important role in many areas such as structural mechanics, thermodynamics, fluid mechanics and electromagnetism.

Finite element method is built on the basis of solid flow variation principle. In the finite element analysis, first, disperse the analyzed objects into many small units; second, set the boundary conditions and the characteristics of load and material; third, solve the linear or nonlinear equations, and get the results such as displacement, stress, strain and internal force; last, show the calculation results with graphics technology on the computer. At present, the common finite element program analysis functions cover almost all the engineering field, and the program is very convenient. The thoughts of analysis and calculation mainly include: 
The object discretization. Discrete an engineering structure into calculation model with various units, and it is called unit classification. After discretion, the units are connected by the nodes; the set, nature and number of unit nodes should be decided by the nature of problems, the need of describing deformation pattern and calculating the progress. Normally, more detailed unit classification can describe the deformation more accurately, namely more closer to actual deformation, but the amount of calculation is larger. So the structure analyzed in finite element is not the original object or structure, it is the discrete object of many units in some way. In this way, the results obtained by finite element analysis calculation are approximate. If the number of divided units is large and reasonable, the obtained result is consistent with actual situation.

The unit feature analysis. Based on the material properties, shape, size, number of nodes, location, and its meaning of units, this paper finds out the relation between the nodal force and node displacement. At this time, establish the equation of force and displacement applying the geometric equation and physics equation of elastic mechanics, and educe element stiffness matrix, which is one of the basic steps of finite element method.

$$
[K] q\}=\{
$$

Unit construction. Using equilibrium conditions and boundary conditions of structure force, reconnect each unit according to the original structure, and form the integral finite element equation:

$[K]\{q\}=\{F\}$

In the above equation: $\mathrm{K}$-stiffness matrix of overall structure

q-node displacement array

F-load array

To solve the unknown node displacement. Get the displacement by solving finite element equation. In the solving process, select appropriate calculation method based on the specific characteristics of equations. According to the above analysis, the basic idea of finite unit method is "detachment-combination"; detachment is for unit analysis, and combination is for the comprehensive analysis on the whole structure. The stress-strain relationship of soil body is mainly about the elastic-plastic problem in the paper, which involves the handling of nonlinear finite element.

Analysis on land subsidence in the tunnel. Pipe jacking construction is a kind of construction method by non-excavating laying underground pipeline, with the aid of the main roof oil tank, pipeline space and intermediate relaying jacking; push tool pipe or road-header into receiving pit from working pit through soil, and bury the pipes following the tool pipe or road-header between the two pits, which requires no excavation surface and can cross roads, railways, rivers, ground buildings, underground structures, all kinds of underground pipeline, etc.

With the development of urban construction, pipe jacking construction has become increasingly popular, and been applied in more and more fields. At present, it has been widely applied in the construction of water pipe, gas pipe, power cable, communication cable and power plant circulating water cooling system. On the basis of pipe jacking, it has been developed into non-excavation construction technology, various non-excavation construction associations and related periodicals are founded.

The stress-strain relationship of soil is nonlinear and complicated, so there are more constitutive models of soil. These models have a great influence on the calculation results; the improper model 
gets worse nonlinear results than linear results and even completely unreasonable. Therefore, the choice of model has decisive significance.

Drucker Prager model reflects the elastic-plastic nonlinear constitutive relation. The adopted yield criterion of Drucker-Prager is the approximation of Mohr-Coulomb criterion, with the purpose of amending the yield criterion of Von-Mises, which means to include addition item in the Von-Mises expression. The yield surface won't change with the gradual yield of material, so there is no hardening rule; however, the yield strength increases with the increase of lateral pressure (hydrostatic pressure), and plastic behavior is assumed as ideal elastic-plastic. In addition, the material model considers the volume expansion due to yield, but considers no the influence of temperature change. Drucker Prager model can be used in 2D and 3D entity unit, can be used in the formula of small displacement - small strain, large displacement-small strain and large displacement-large strain. Some of the important characteristics of Drucker Prager model are shown in Fig. 1.

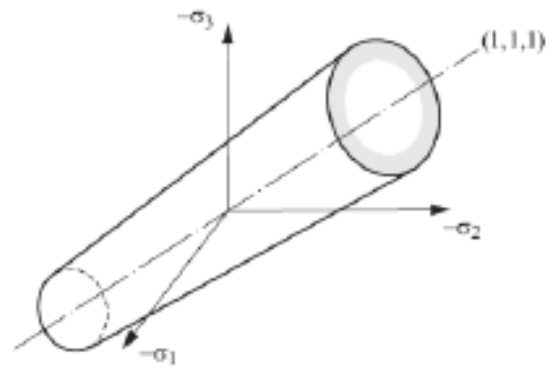

Fig. 1 DP Yield Model in Principal Stress Space

The yield criterion of Drucker-Prager is shown in the following formula:

$$
f_{D P}^{t}=\alpha^{t} J_{1}+\sqrt{J_{2 D}^{t}}-k
$$

In the formula, $\alpha, \mathrm{k} —$ characteristic parameter

$$
J_{1} \_ \text {first invariant of stress at the moment of } \mathrm{t}
$$

$$
J^{t}{ }_{D P} \_ \text {second invariant of deviatoric stress at the moment of } \mathrm{t}
$$

Numerical calculation and simulation of land subsidence. Many cities in our country is located in soft soil area; these types of soft soil include slit, slit clay, silty clay and sand, which have the features of rich water content, bigger hole and high compressibility, and are caused by lagoon facies, drowned valley facies, lakeshore facies, delta facies, limnetic facies, river and flood plain facies, drift bed and diluvial facies. On this basis, select the following representative soil for modeling.

On the problem of seepage and drainage, ADINA needs to input the permeability of porous medium. Permeability is the constant of permeability for porous medium, only related with the nature of the porous media itself, usually shown with $\mathrm{k}$, and its unit is $\mathrm{m}^{2}$; Permeability coefficient is commonly shown with $\mathrm{K}$, and its unit is $\mathrm{m} / \mathrm{s}$, which has the following relations with permeability $\mathrm{K}$ :

$$
K=\rho g / \mu
$$

in the above formula, $\rho$ _ the density of liquid, also the density of water in the calculation, with the unit $\mathrm{kg} / \mathrm{m}^{3}$; 
$\mathrm{g}$ - the gravity acceleration, with the unit $\mathrm{m} / \mathrm{s}$;

$\mu$ - liquid coefficient of kinetic viscosity, with the unit $\mathrm{kg} / \mathrm{m} \cdot \mathrm{s}, \mu$ of water with the temperature of $25^{\circ}$ is $0.001 \mathrm{~kg} / \mathrm{m} \cdot \mathrm{s}$.

Generally, get the permeability coefficient $\mathrm{K}$ of soil in the data, and $\mathrm{k}$ needed for ADINA program through calculation. In engineering calculation, the unit of pressure and modulus is $\mathrm{Pa}$, and $\mathrm{s}$ is the unit of time; therefore, $\mathrm{k}$ is the value of $\mathrm{K}($ unit: $\mathrm{m} / \mathrm{d}$ ) divided by 10 , that is the gravity acceleration of water; namely, $\mathrm{k}=$ permeability coefficient/10.

In ADINA, the constitutive models on soil are Cam Clay model, Mohr - Coulomb model, and Drucker Prager model. In addition, develop the Duncan-Chang model of ADINA by making use of the characteristic "user-defined material constitutive" contained in ADINA and calculate.

(1) According to the scope of the simulation, establish finite element model, and define the relevant boundary conditions, gravity load and material properties of soil, etc.

(2) Before the lining unit are "activated", calculate the subsidence of model under the condition of the self-weight of soil, consider the existing subsidence of soil before the excavation of tunnel, which means to eliminate the influence on land subsidence from early history;

(3) "Activate" lining unit, and calculate the land subsidence due to soil loss after the tunnel is excavated. The so-called unit activation is to calculate the unit stiffness matrix, mass and load of column according to the properties of the lining material. Among them, make use of the repetitive unit existed in ADIN A supportive model (i.e., the same position with different material unit, different nodes, or different material unit at the same position and same nodes, etc), and deal with the simulation of complicated excavation supporting process;

(4) Analyze and study the results by using post-process program

\section{Conclusions}

The paper studied relevant theories of land subsidence caused by tunnel excavation at home and abroad. Combined with related theories such as the soil constitutive model and finite element, the paper constructed the finite element model by adopting ADINA nonlinear analysis software. Based on the finite element model, it simulates the land subsidence caused by tunnel excavation through numerical simulation, studies related factors affecting land subsidence of tunnel, makes comparative analysis of the main influencing factors of tunnel land subsidence, and comes to the following conclusions:

(1) Describe in detail how to simulate tunnel excavation using ADINA, show the computing method of land subsidence caused by tunnel excavation using the software, and how to simulate the influence on ground of soil excavation using birth-death unit computing method; establish simulated finite element model, and show corresponding calculating width, parameter setting, boundary conditions setting, and calculation steps, which provide convenience for later numerical analysis to some extent.

(2) In the choice of the constitutive model of soil, make numerical contrast calculation by adopting Drucker Prager model, Mohr-Coulomb model, Cam Clay model and the secondary development Duncan - Chang model; compare the four calculation results with theoretical results; if the results adopting Mohr - Coulomb model are close to the theoretical results, it shows that using this model in ADINA is fit for simulation of land subsidence caused by tunnel excavation.

(3) Compare the settlement trough curves in drained with the undrained condition, and the results show that in any case, the surface settlement troughs are approximate to the normal distribution 
curve, but the result of the drainage conditions is larger than the undrained conditions, and there is wide range of ground impact; however, the difference value of land subsidence at the same point drops by some percentage when gradually get away from the tunnel center.

In recent years, the research on land subsidence caused by tunnel excavation has made big progress, but there are still many practical engineering problems to be solved because of the complicated soil constitutive relationship and the complexity of the project. In the later research work, the following several aspects should be studied in depth:

(1) Considering the influence of anisotropy of soil, visco-elastoplastic, seepage and drainage, establish the model more in line with the soil stress-strain relationship, so as to build more reasonable finite element analysis model by finite element software.

(2) Although the 2D plane strain finite element analysis adopted in this paper can better simulate the land subsidence caused by tunnel excavation, the tunnel excavation has $3 \mathrm{D}$ characteristics in practical engineering. It is quite necessary to establish a 3D finite element model, and the 3D model that is more in line with the actual situation can make numerical analysis on the land subsidence caused by tunnel excavation.

\section{Acknowledgment}

This work was supported by Project on professional and characteristical construction of Jiangxi province 2010 (Civil Engineering) and Project on the planning and construction of disciplines in Jiangxi University of Technology (Structure Engineering)

\section{References}

[1] Minqiang W, Shenghong C. 3-DIMENSIONAL NON-LINEAR FINITE ELEMENT SIMULATION OF TUNNEL STRUCTURE FOR MOVING-FORWARD SHIELD [J][J]. Chinese Journal of Rock Mechanics and Engineering, 2002, 2: 017.

[2] Phien-Wej N, Giao P H, Nutalaya P. Land subsidence in Bangkok, Thailand[J]. Engineering Geology, 2006, 82(4): 187-201.

[3] Xu Y S, Ma L, Du Y J, et al. Analysis of urbanisation-induced land subsidence in Shanghai[J]. Natural hazards, 2012, 63(2): 1255-1267.

[4] Wang T, Liu W, Zhang C, et al. Study on ground settlement induced by shallow metro station constructions $[\mathrm{J}]$. Yanshilixue Yu Gongcheng Xuebao/Chinese Journal of Rock Mechanics and Engineering, 2007, 26(9): 1855-1861.

[5] Peng F L, Wang H L, Tan Y, et al. Field measurements and finite-element method simulation of a tunnel shaft constructed by pneumatic caisson method in Shanghai soft ground[J]. Journal of Geotechnical and Geoenvironmental Engineering, 2010, 137(5): 516-524.

[6] Tang Y, Zhou J, Ren X, et al. Introduction[M]//Dynamic Response and Deformation Characteristic of Saturated Soft Clay under Subway Vehicle Loading. Springer Berlin Heidelberg, 2014: 1-24.

[7] Peila D. A theoretical study of reinforcement influence on the stability of a tunnel face[J]. Geotechnical \& Geological Engineering, 1994, 12(3): 145-168.

[8] Liang W, Mingli H. Subsidence and deformation control of large-span tunnel in water-bearing 
unfavorable geological conditions with CRD method[J]. Chinese Journal of Rock Mechanics and Engineering, 2007, 26(3): 738-3.

[9] Yang H, Fu D, Ge X. Experimental study and numerical simulation of earth pressure around shield machine[J]. Yanshilixue Yu Gongcheng Xuebao/Chinese Journal of Rock Mechanics and Engineering, 2006, 25(8): 1652-1657.

[10] Jin X R, Yu J L, Zhu S C, et al. Analysis of behaviors of settlement of pit's surrounding soils by dewatering[J]. Yantu Lixue(Rock and Soil Mechanics), 2005, 26(10): 1575-1581. 